\title{
Qualitative Physics in a Metaphysical Perspective
}

\author{
Aleksandr Kulieshov ${ }^{1}$ \\ ${ }^{1}$ Cherkasy State Technological University \\ 460 Shevchenko Boulevard, 18006, Cherkasy, Ukraine
}

DOI: $10.22178 /$ pos.44-5

LCC Subject Category: BD95-

131

Received 20.02.2019

Accepted 20.03.2019

Published online 31.03.2019

Corresponding Author:

klshv2014@gmail.com

(C) 2018 The Author. This

article is licensed under a

Creative Commons

Attribution 4.0 License

(c) (1)
Abstract. The article deals with the problem concerning the possibility of qualitative physics paradigm development and its close connection with metaphysics. The idea of qualitative physics is based on the principles of Aristotelian physics and is opposed to quantitative modern physics (classical and non-classical). It is stated that the essential difference between the two physical paradigms lies in the ways of describing physical objects. Qualitative physics presuppose the qualitative description of physical objects independent of their quantitative description. In normal nowadays physics, on the contrary, physical objects are regarded to be fully determined through quantitative (numerical and structural-analytical) relationships with other objects. The statements of modern physics are considered reasonable if they can be self-consistently expressed by the apparatus of mathematics. The article shows that this way of describing and explaining physical reality is incomplete. There is ground to assert that the quantitative relations of physical objects do not encompass everything that exists in the relations of physical objects. It is argued that there are qualitative aspects of physical reality that are not defined quantitatively and may become the content of special qualitative physics. The conclusion is made that such qualitative physics in its principles and language must be close to traditional metaphysics and can appear to be an application of metaphysics to the field of physical reality.

Keywords: physical paradigm; qualitative physics; quantitative physics; metaphysics; the way of describing physical objects.

\section{INTRODUCTION}

The term "qualitative physics" has nowadays at least two spheres of application. One concerns a branch of physical research connected with artificial intelligence having been formed in USA in 80-90-ies of the 20th century. It is characterized by the general understanding of qualitative physics as a set of methods which can help in achieving some results within physical science. In [4] this research branch is depicted in such a way: "In most qualitative physics approaches, systems are characterized in terms of parameters that take on "qualitative values". Such qualitative values may be obtained from the respective quantitative (i. e. real-valued) description by considering significant "landmarks" which discriminate qualitatively between different states or modes of behaviour (e. g. the freezing point and the boiling point of a fluid object, or the maximal opening of a valve) [4, p. 124]. Thus, qualitative physics is reduced to a set of qualitative methods applied to objects that are differently described quantitatively. The peculiarity of qualitative methods consists in taking into account only qualitative parameters. "As a matter of fact, one important goal of qualitative physics is to support reasoning about physical systems that does not require (or pretend to have) exact numerical information about them" [4, p. 124]. Qualitative physics understood in this way does not preclude the formalization of qualitative parameters, their expression in mathematical form (in the form of a special algebra), which should facilitate the transfer of qualitative physical reasoning to the level of machine intelligence. As it was stated by K. D. Forbus, "Qualitative physics arises from the need to share our intuitions about the physical world with our machines" [5, p. 241]. In general, such qualitative physics does not differ from mathematical physics by its objects, goals, and general principles of research. It differs only in specified parameters and in methods of operating with them. Qualitative physics refines and 
complements the results obtained by using quantitative analysis, and in this sense, qualitative physics remains entirely within the science of physics as it is now understood. Experts in the field of qualitative physics agree in viewing "qualitative physics as an extension of existing scientific disciplines rather than as an entirely new field of endeavour" [8, p. 19].

Another interpretation of qualitative physics is present in the historical analysis of early physical theories, especially ancient ones. First of all, this term refers to the physics of Aristotle. Qualitative physics means here not one of the aspects of physical research, but physics in general. This physics appears to be an alternative to classical and modern physics which is mathematized and based on experiment and measurement. At the same time, Aristotle's physics is closely connected with metaphysics, is an application of metaphysics to the field of physical phenomena. I. Bodnar notes that “Aristotle's metaphysics and physics use a common conceptual framework, and they often address similar issues. The prime and distinctive task of first philosophy is an inquiry into first entities; these, however, are not perceptible entities, and as a result they have to be investigated through a metaphysical investigation of physical entities. Hence the overlap between the two disciplines, which often verges on inseparability" [1]. Aristotle's Physics, as C. Shields notes, "is not, of course, a work of the sort we find in modern quantitative physics. It is, rather, a puzzle-driven inquiry into features and facets of nature at their most general level" [10, p.196]. Aristotle's physics is qualitative in two ways. First, we are talking about a qualitative understanding of the empirical basis of science. Perceived qualities underlie the reasoning in the physics of Aristotle. According to M. Mouzala, "The crucial point of Aristotle's theory of method in the Physics I.1 is that perception, the only faculty of human being that can have access to the composite natural thing, naturally grasps it as an indiscriminate, inarticulate and confused whole" $[6$, p. 48]. The purpose of the analysis in Aristotle's physics is to explain the qualitative transformations to which the quantitative ones are also reduced (increase and decrease in quantity, movement).

More importantly, another feature of Aristotle's qualitative physics is that it lacks mathematical apparatus as a means of expression and a way of grounding its statements. This aspect of Aristotle's physics does not escape researchers' attention. J. Schummer argues that "for Aristotle and his followers, mathematics was clearly distinct from physics, because it only described nature in geometrical or numerical terms. The task of physics was, however, to explain nature" [9, p. 760]. The absence of the mathematical form of expression means that in the explanation of physical objects their measurable (homogeneous) attributes are not highlighted, these attributes are not formalized in the form of variables and constants. In addition, numerical or analytical (in terms of sets and their elements) relations between selected physical objects are not established. In this respect, the concept of qualitative physics is opposed to the concept of not just quantitative, but mathematized physics. It seems like this is the main sense of qualitative physics. Qualitative physics is the physics without mathematics, serving at least as the background for physical research. This is the physics without measurements or descriptive physics, which is reduced to the selection of qualitative differences in physical reality.

It is established that the transition from Aristotelian physics to modern one occurred in the 17th century. And although there are doubts about the chronological framework of this process [7], the general shift from qualitative physics to mathematized one is obvious. The principle idea of the "grand narrative" (as it is called by S. Roux) about the seventeenth century Scientific Revolution is that mathematization should be taken "as the criterion for distinguishing between a qualitative Aristotelian philosophy and the new quantitative physics" [7, p. 320]. It may be that the introduction of mathematics in natural philosophy had been more gradual and less revolutionary during the Renaissance and the Early Modern period but it cannot be denied that "for Aristotle, mathematics merely captures the superficial properties of things" and this position is vastly different from the modern physics. Classical physics had emerged to the great extent by overcoming the flaws of qualitative physics. A new understanding of empirical basis had appeared. It consisted in the formalized observation and experiments methodically subordinate to the process of measuring physical quantities and their relations. But even more important thing - principally important for all modern physics, from the 17th century to the present day - was the conviction ex- 
pressed at the time by Albert Einstein: "Our experience up to date justifies us in feeling sure that in Nature is actualized the ideal of mathematical simplicity. It is my conviction that pure mathematical construction enables us to discover the concepts and the laws connecting them which give us the key to the understanding of the phenomena of Nature. Experience can of course guide us in our choice of serviceable mathematical concepts; it cannot possibly be the source from which they are derived; experience of course remains the sole criterion of the serviceability of a mathematical construction for physics, but the truly creative principle resides in mathematics" [3, p. 167]. It should be added that the new, non-Aristotelian and, in this sense, nonqualitative physics also presupposed its separation from metaphysics, along with reliance on one's own capabilities in solving fundamental problems.

The physics of nowadays looks like a set of formulas, at least it can be most fully expressed in this way. Physical entities are defined by their formal (quantitative) relations to other entities. The qualitative parameters are derived from numerical-analytical. By virtue of this, a qualitative description is embedded in a quantitative one and cannot be an alternative to it. Physics is an external area of knowledge for metaphysics (in general - for philosophy). Moreover, in a dispute about who will establish the ultimate truth regarding the universe, physics looks more convincing.

This state of affairs causes concern among philosophers and, in part, among physicists themselves. Some paradigmatic problems of modern physics and its impact on physics-metaphysics relation can be found in materials of Scientific Models and a Comprehensive Picture of Reality workshop, arranged by the Finnish Society for natural philosophy together with the physics Foundations Society (Helsinki, 2016). One of conclusions made is that today`s physical theories obtain the role of mathematical descriptions of observations instead of serving as real building blocks for a comprehensive picture of reality [11, p. 7].

Nevertheless in modern physics, the history and the philosophy of physics, the attitude towards qualitative physics as a special physical paradigm remains in general negative. Aristotelian physics is estimated as a dead-end branch of the devel- opment of science. Here is one of the characteristic estimates for the work on the history of ancient physics, giving preference to the physics of Democritus over the physics of Aristotle: "...classical atomism was far superior to the physics of Aristotle, which upheld real qualitative differences between the four - or rather five - heterogeneous elements and which, in regarding, for instance, the process of evaporation as a real transformation of one element - water - into another - air - also believed in real qualitative changes in nature. The modern concept of matter as homogeneous stuff differentiated only by the quantitative differences between the ultimate particles - that is, their size, shape, position, and motion - was fully anticipated by Democritus while it remained completely foreign to Aristotle`s qualitative physics. ...There is no question now which of these views was superior and which was closer to the spirit and even to the letter of Newtonian physics [2, p. 4-5]. So, Aristotle's physics is an alternative physical paradigm - this is recognized. However, this paradigm is fundamentally wrong (it can be put in this sense next to the Ptolemy astronomical paradigm). A more general inference is also legitimate here: qualitative physics is completely archaic and does not deserve attention. It is advisable not to take it into account in modern thinking about the world. Though we can accept it in another sense indicated above - as a set of methods complementing the basic physical research.

The objective of the article is to show that such a complete negation of qualitative physics as an alternative physical paradigm is excessive. There are limitations and uncertainties in modern mathematized physics, which make it impossible to present a complete picture of reality.

It will be argued, that there is the possibility to have a qualitative point of view on physical reality, which is to some extent an alternative view of things and which may fill those gaps in the picture of reality that are inevitable when explaining the world by means of mathematized physics. This is, of course, not the restoration of Aristotle's physics in the form in which it existed. One may talk only about the efficiency of the very principle of qualitative physics as a complementary alternative to mathematized physics. It will also be demonstrated that in addition to the quality principle itself, such physics is similar to the Aristotelian one in its closer connection with metaphysics. 


\section{RESULT AND DISCUSSION}

Almost all objects of modern physics are described by mathematical formulas. They are being installed by numerical relations as well as structural-analytical relations (relations of inclusion) with other objects. A physical object exists in a physical theory as a side or a part of a certain equation (or rather equations). Other parts of the equation are defined similarly. Thus, the complete definition of a physical object is reduced to a set of values (variables and constants) expressed eventually through this object. Like any mathematical theory, modern physics is implicitly tautological. The basic concepts in it refer not to actual physical objects, but to the meanings of elementary mathematical expressions.

As an example we can take the concept of the mass of a physical body. In classical physics, the inertial mass is determined through external force and acceleration, and the gravitational mass through the force of gravity and gravitational acceleration. In its turn, force is determined through mass and acceleration. The force of gravity is determined through the mass and acceleration of free fall or through other values, including the gravitational constant and the distance between the centres of mass of attracted bodies. The distance can be determined through acceleration and time, time - through the path length and the average speed, which, in turn, is determined through acceleration, etc. All values are interrelated or related to values that are constants. The extension of classical physics to relativistic is carried out through the complication of mathematical apparatus. In relativistic mechanics, mass is determined through the total energy of a body, its momentum (the product of mass and velocity) and the speed of light. Each of the objects determining mass, in turn, is determined through other values, indirectly including mass. Thus, the relativistic mass is a side of the ratio of quantities and does not differ from other quantities as a quantity (in other words, a mathematical object).

Physical objects in another basic theory of modern physics - quantum mechanics - are determined in a similar way. The specificity of quantum mechanics is a much greater complexity of relations, including the use of such branches of mathematics as statistical mathematics and probability theory and the supplementation of relations with new constants (in particular, the Planck constant). The nature of correlated values as being defined by their numerical or structuralanalytical relations does not change. New theories in physics of the late twentieth - early twenty-first century are also introduced through the complication of mathematical apparatus, which does not change the nature of the relations defining physical objects. Thus, the now popular hypothesis of fundamental physics - string theory - involves one-dimensional (in some cases, multidimensional) spatial objects, defined by space-time parameters, which, in turn, are determined by their movement. It seems that any objects detected by physics are not defined otherwise than by quantitative (numericalanalytical) relationships with other objects defined in the same way.

Do the quantitative (further we will use this term in a broad sense - that is, non-qualitative) definitions exhaust the essence of physical objects? The answer to this question must be negative. Quantitative definitions express exclusively relationships, but relationships cannot be only relationships. In relationships, there is always something that relates (relata). Even if the relata of a relationship are determined by the relationship itself, they are not identical to the relationship itself. In relata there is something that makes them the related sides of a relationship, and not the relationship itself, something that distinguishes them from the relationship as such. In other words, something must acquire some form in specific relationship and this substratum in no case is quantitative. When we define the parameters of a physical object, we define the parameters of something which is out of all parameters. It is impossible for pure quantities or pure mathematical objects to exist in material physical reality. This is impossible by definition (physical reality is not identical to a mathematically constructed reality). If all physical objects have quantitative definitions, then this means that there is something and there are quantitative definitions of it. Obviously, this something eludes quantitative physics.

In classical and relativistic physics it is customary to talk about body mass. Mass is defined by its relations with other quantities. But what is a body? Outside the mass (the quantitative parameter determined by other quantitative parameters) it turns out to be just a part of the space associated with the mass parameter. However, a part of the space as such is not necessarily connected with mass, it needs further definition applying the factor that remains indefinable in 
quantitative physics. A physical body must have some entity besides all its quantitative and spatial definitions. This concerns not only to the concept of a physical body. What is a string in string theory? It is a one-dimensional physical object that changes its position in multidimensional space. One-dimensionality, multidimensionality, spatial position are quantitative determinants (given by the corresponding formulas). In addition to them, there is a physical object which has quantitative definitions. The concept of a physical object is empty. We do not know what exactly is designated by this term. It is unlikely that researchers engaged in string theory can answer the question of what exactly is that is onedimensional and fluctuating in their theory. On the other hand, it is impossible to identify a physical object with its properties, in this case with one-dimensionality and oscillatory motion. One-dimensionality and oscillatory motion cannot be identified with a physical object, they require some substratum. What is such a substratum? Nothing or something?

The question of a substrate existence appeals to metaphysics and is the subject of discussion. There are metaphysical concepts (so called "bundle theories") stating that the totality of qualities exhausts any existing object. This view does not exclude the existence of a substrate or rather something which performs the function of a substrate. The substrate can be defined as the quality that is identical with combining or linking a number of other qualities. In this case, it would be possible to adopt the viewpoint that there are neutral physical objects (substrates) completely determined by their quantitative relations with other objects. The notion of a physical object thus refers to the ability to be determined by concrete quantitative relationships.

But this assumption does not explain the fact that physical objects are different. Let's assume that a physical object is identical with the ability to be determined by some quantitative relations. A question should be asked: is this ability the same for all physical objects or are objects different in this ability? If the ability is the same, then all physical objects must also be the same (at least qualitatively). Such a conclusion contradicts the empirical data we have. If this ability is different for different physical objects, then there must be some ground for this. And this ground must be in the objects themselves, which makes them different initially, before any quantitative relations they are in. Without admitting such a state of af- fairs it is impossible to explain variety of physical objects quantitative relationships with other objects.

It follows that in addition to any quantitative distinctness, physical objects have some qualitative differences. It is reasonable to think that these qualitative differences are not determined by the quantitative relations of physical objects. In other words, physical objects outside their quantitative definitions are not qualitatively neutral. This is explained by the fact that qualitatively neutral physical objects cannot be different, they are all the same. There is, therefore, some initial (relative to quantitative relations) qualitative distinctness of physical objects. Whether such qualitative distinctness is fundamental to all that exists is a metaphysical and debatable question. Rather, it may be assumed that the primary qualities of physical objects are given by their special qualitative relations with other physical objects. There is also reason to think that, in general, the defining qualitative relationships are asymmetric and represent grounding relationships. One may add that the types and ranges of values of the physical objects' quantitative relations are determined by their qualitative differences.

The peculiarity of the qualitative description of physical objects is that such a description does not require an answer to the question: what is the carrier of qualities. Qualities are identifiable with physical objects, whereas quantitative parameters are identifiable with relations of objects. You can separate quantitative relationships from objects, leaving objects essentially identical but you cannot separate qualities (meaning primary, substrate qualities or attributes) from objects without destroying the objects themselves. Thus, it is plausible that the qualities of the substrate of other qualities and of quantitative relations are identical with the substrate itself. This removes the question of the carrier of qualities being separate from all qualities. Substrate qualities of specific objects can be generated by other qualities identical to other substrates, which, in the end, leads to fundamental qualities identical to the primary ungrounded substrate or fundamental reality. Such a picture of the world is hypothetical, but at least consistent.

Qualitative description requires an appropriate terminological apparatus. It looks obvious that at least some of the terms referring to objects that differ qualitatively, regardless of their quantita- 
tive parameters, should differ from the terms of quantitative physics. Conversely, not all the terms of quantitative physics should be applicable in the qualitative description. The rules for joining terms and the signs of relations between them may differ. So the description of purely qualitative physical objects needs a special language. It seems not ungrounded to talk about qualitative description as a different physical discipline, qualitative physics.

Of course, there are doubts. Isn't the project of qualitative physics an attempt to multiply entities without need? Shouldn't Occam's razor be used here? It is customary to think that physical reality is one. What will be given to us by its description, based on different principles? Quantitative physics demonstrates the ability to show what exists in the physical world. It also exhibits the way physical objects interact, how they move, points out to the immediate causes of physical phenomena. This result obtaining is sufficient to create a plausible picture of physical reality, to have some theoretical understanding of the physical world and to use it for practical purposes.

The sense of qualitative physics, if such physics is ever to arise, will be the explanation of why certain physical objects exist at all. Qualitative physics should be engaged in figuring out how certain physical events and processes are possible in the physical world, defining what can and what cannot physically exist, and in particular what does not exist in the physical world, but could exist. Without the implementation of this research program, it seems that a complete understanding of physical reality is impossible.

It is clear that qualitative and quantitative physics must be interconnected. In quantitative physics, the validity of fundamental physical concepts, as a rule, boils down to the consistency of their mathematical models. There may be more than one such models. Qualitative physics could provide decisive arguments for choosing one or another interpretation of quantitative physical data.

There is no doubt that qualitative physics objectives in general coincide with the goals of metaphysics. The metaphysics in its traditional form has always been searching for the same as it would be natural of qualitative physics - to find out how it is possible for something to exists in the world. At the same time, metaphysics endeavours to represent that which exists with utmost completeness and from an extremely ab- stract point of view, by expanding its search up to elementary entities (we do not here solve the metaphysical question of whether these entities should be simple or complex). It is clear that in this sense, qualitative physics is inferior to metaphysics in scope and level of abstractness.

The language of metaphysics, as well as the language of supposed qualitative physics, is qualitative. It is the language reflecting reality prior to its quantitative differences. It can be called the language of differences as such. This homogeneity of the languages of metaphysics and qualitative physics suggests that the terms of qualitative physics could constitute a sublanguage of the language of metaphysics. In the absence of specific theories of qualitative physics, it is now difficult to establish more precisely the relationship between the two disciplinary languages.

In general, it is obvious that metaphysics and the alleged qualitative physics compose a fundamental unity. At that, qualitative physics directly follows from metaphysics and is based on it. Perhaps it would be permissible to speak of qualitative physics as an applied metaphysics in the field of physical reality. Or about borderline discipline on the verge of metaphysics and physics. In any case, such a project would revive the unity of metaphysics and physics, from which Aristotle proceeded.

\section{CONCLUSION}

The two most fundamental paradigms are distinguishable in the history of physics. The first is the paradigm of Aristotle. Aristotelian physics is organically connected with metaphysics and based on a qualitative description of physical objects. The other is the paradigm of modern physics (classical and non-classical), formed around the 17 th century. The physics of this paradigm can be called quantitative. Physical objects in it are determined through quantitative (numerical and structural-analytical) relationships with other objects. The statements of modern physics are considered reasonable if they can be selfconsistently expressed by the apparatus of mathematics. The article shows that this way of describing and explaining physical reality is incomplete.

There is ground to assert that the quantitative relations of physical objects do not encompass everything that exists in the relations of physical objects. So there are qualitative aspects of physi- 
cal reality that are not defined quantitatively. They require a separate qualitative description, independent of a quantitative (mathematized) one. Such a description can shape the content of qualitative physics paradigmatically different from modern theoretical physics. The qualitative physics in its principles and language must be close to traditional metaphysics and can appear to be an application of metaphysics to the field of physical reality. This corresponds to the initial physical paradigm of Aristotle.

\section{REFERENCES}

1. Bodnar, I. (2018). Aristotle's Natural Philosophy. In E. Zalta (Ed.). The Stanford Encyclopedia of Philosophy (Spring 2018 ed.). Retrieved from https://plato.stanford.edu/entries/aristotlenatphil/

2. Cohen, R., \& Wartofsky, M. (1983). Physical Sciences and History of Physics. Dordrecht: Springer.

3. Einstein, A. (1934). On the Method of Theoretical Physics. Philosophy of Science, 1(2), 163-169.

4. Faltings, B., \& Struss, P. (Eds.). (1992). Recent Advances in Qualitative Physics. Cambridge: The MIT Press.

5. Forbus, K. D. (1988). Qualitative physics: Past, present, and future. In H. Shrobe, Exploring Artificial Intelligence (pp. 239-296). San Mateo: Morgan Kaufmann Publishers Inc.

6. Mouzala, M. G. (2013). Aristotle's Method of Understanding the First Principles of Natural Things in the Physics I.1. Peitho. Examina Antiqua, 1(3), 31-50. doi: 10.14746/pea.2012.1.2

7. Roux, S. (2010). Forms of Mathematization (14th-17th Centuries). Early Science and Medicine, 15(4), 319-337. doi: 10.1163/157338210x516242

8. Sacks, E. P., \& Doyle, J. (1992). Prolegomena to any future qualitative physics. Computational Intelligence, 8(2), 187-209. doi: 10.1111/j.1467-8640.1992.tb00344.x

9. Schummer, J. (2008). Aristotelian Physics. In K. L. Lerner \& B. W. Lerner (Hg.), Scientific Thought in Context (Vol. 2, pp. 759-768). Detroit: Gale.

10. Shields, C. (2007). Aristotle. London: Routledge.

11. Suntola, T., \& Styrman, A. (Eds.). (2016). Scientific Models and a Comprehensive Picture of Reality. Rome: La Nuova Critica. 